Ann. Biol. anim. Bioch. Biophys., I972, 12 (3), 397-409.

\title{
RÉPONSE FOLLICULAIRE DU VEAU FEMELLE IMPUBÈRE A DIVERS TRAITEMENTS DE GONADOTROPINE SÉRIQUE ASSOCIÉE OU NON A UN PROGESTAGÈNE
}

\author{
J. TESTART \\ avec la collaboration technique de F. Aymer de la Chevalerie \\ Station centrale de Physiologie animale, \\ Centre national de Recherches zootechniques, I. N. R. A., \\ 78350 - Jouy-en-Josas

\section{RÉSUMÉ}

A partir de coupes sériées d'ovaires de Veau impubère, de $250 \mu$ d'épaisseur, obtenues au cryostat, on a mesuré et compté les follicules de diamètre au moins égal à $\mathrm{I} \mathbf{m m}$, après divers traitements hormonaux.

La méthode de dénombrement utilisée est rapide et fait apparaître $30 \mathrm{p}$. Ioo de follicules supplémentaires par rapport à l'examen des ovaires in toto; la population folliculaire, d'une grande variabilité individuelle, n'est pas différente entre les deux ovaires.

La croissance folliculaire suivant l'injection i. v. de 2 ooo UI de PMSG (exp. A) ou suivant les injections $i . v$, de I25 UI de PMSG tous les 2 jours (exp. B) est d'abord sensible sur les follicules de $4 \mathrm{~mm}$.

Les follicules de 4 à $5 \mathrm{~mm}$ constituent la population critique dont l'effectif s'accroît 16 heures (exp. A) ou 8 jours (exp. B) après le début du traitement, aux dépens des follicules de 2 à $3 \mathrm{~mm}$ qui constituent la population de stockage.

On obtient l'ovulation des follicules, stimulés par PMSG seule, après $72 \mathrm{~h}(\exp . \mathrm{A})$ ou yo jours (exp. B), mais seul le traitement prolongé (exp. B) permet la différenciation de corps jaunes normaux (16 à $25 \mathrm{~mm}$ ) et une croissance de l'utérus de roo p. roo.

La pose d'une éponge vaginale imprégnée de $60 \mathrm{mg}$ de FGA, en début de traitement long par PMSG (exp. B-FGA) prévient toute évolution folliculaire jusqu'à to jours, puis la croissance accélérée de quelques follicules.

\section{INTRODUCTION}

La présence de follicules à antrum dans l'ovaire du veau dès la naissance (CASIDA et al., I935; MARDEN, I953) et la constatation que leur nombre maximum est atteint avant la puberté (ERICKSON, I966) expliquent les nombreuses tentatives faites pour obtenir la maturité folliculaire et 1'ovulation chez le veau femelle impubère (CASIDA, I943; MARDEN, I953; BLACK et al., I953).

Des taux d'ovulation importants ont été obtenus en faisant suivre un traite- 
ment folliculotrope (PMSG ou FSH) d'une injection d'HCG (LINEWEAVER et HAFEZ, I970; HAFEZ, I970) ou de LH (JAINUDEEN, LINEWEAVER et HAFEZ, I966 ; AVERY et al., I962 ; ONUMA et FoOTE, I969; ONUMA et al., I969, I970 ; SEIDEL, LARSON et Foo'te, I97I), mais la plupart des auteurs constatent une asynchronie dans les ovulations; ONUMA et Foore (I969) l'attribuent à la rupture plus rapide d'un gros follicule préexistant sur l'ovaire. En outre, les réponses sont extrêmement variables d'un individu à l'autre, témoignant de la présence dans l'ovaire d'un nombre différent de follicules à antrum susceptibles d'être stimulés par la gonadotropine, ou d'une sensibilité variable des animaux, bien mise en évidence par MauLÉoN et al. (I970) chez la Vache. D'autres éléments peuvent également modifier la réponse : celle-ci passerait par un maximum à 2-3 mois (HOWE et al., I962 ; SEIDEL et FoOTE, I969) ou à 5 mois (SEIDEL, LARSON et Foote, I97I) ; par ailleurs, YING et MEYER (r969) montrent que la réponse ovulante de la Rate immature à des doses croissantes de PMSG n'est pas linéaire mais présente 2 pics correspondant à 3 et 30 UI de PMSG.

Pour essayer de comprendre ces variations dans les réponses de l'ovaire, nous avons cherché à connaître la croissance des follicules à antrum sous 1'influence de la PMSG, en présence ou non d'un progestagène, ce dernier étant destiné à freiner l'excrétion de base et une décharge ovulante éventuelle de gonadotropines endogènes.

\section{MATÉRIEL ET MÉTHODES}

\section{A. - Technique d'étude}

Pour l'analyse de la cinétique folliculaire, la méthode employée par Pedersen (1970) chez la Souris, comportant l'étude des ovaires par coupes sériées de $5 \mu$ d'épaisseur, après marquage radio-actif, est difficile chez le Veau pour des raisons économiques. De plus, l'analyse de coupes fines sériées représente un travail considérable même si, comme Rajakoski (1960), on effectue des coupes de ro $\mu$ tous les $200 \mu$. Aussi, avons-nous procédé par coupes sériées épaisses (250 $\mu$ ) obtenues au cryostat; cette technique ne permet que l'examen et la mensuration du follicule, ovocyte et granulosa disparaissant de l'antrum lors de la décongélation.

Après l'abattage, le tractus génital était prélevé et disséqué. Les ovaires étaient examinés et leur volume mesuré avant congélation à $-\mathrm{I}^{\circ} \mathrm{C}$; 1'utérus, isolé du cervix et des trompes, était pesé.

L'état de développement des plus gros follicules, apprécié d'abord par examen de l'ovaire in toto était précisé par l'étude des coupes sériées.

Ces coupes, étalées sur lame, étaient colorées au bleu de méthylène à I p. roo (pour mieux faire apparaître les cavités folliculaires) et montées par trempage dans la résine "Afcolène " (Péchiney-Saint-Grobain).

Sous loupe binoculaire, on a mesuré les deux plus grands diamètres perpendiculaires, à partir du bord intérieur de la thèque externe, de chaque follicule, et on a calculé leur moyenne arithmétique. Seuls les follicules d'au moins I $\mathrm{mm}$ de diamètre étaient recensés.

Soit, $2 \mathrm{R}$, le diamètre réel du follicule, $2 \mathrm{R}^{\prime}$, le diamètre lu, $e$, l'épaisseur des coupes $\left(25^{\circ} \mu\right)$.

On peut calculer l'erreur maximum par défaut commise dans l'appréciation du diamètre, en fonction de la taille du follicule, sachant que $\mathrm{R}^{\prime}=\sqrt{\mathrm{R}^{2}-e^{2}}$

$\mathrm{Si}$

$$
\begin{array}{ll}
\mathrm{R}=0,5 & e=\frac{\mathrm{R}}{2} \text { et } \mathrm{R}^{\prime}=0,433 \\
\mathrm{R}=\mathrm{I} & e=\frac{\mathrm{R}}{4} \text { et } \mathrm{R}^{\prime}=0,968 \\
\mathrm{R}=2 & e=\frac{\mathrm{R}}{8} \text { et } \mathrm{R}^{\prime}=\mathrm{I}, 984,
\end{array}
$$

etc. 
Ainsi, l'erreur maximum par défaut, qui est de I3,4 p. Ioo pour les follicules de I mm, n'est plus que de 3,2 p. roo pour les follicules de $2 \mathrm{~mm}$ et seulement $0,8 \mathrm{p}$. Ioopour les follicules de $4 \mathrm{~mm}$. Cette erreur est toutefois augmentée de celle commise en négligeant l'épaisseur de la thèque externe, son bord interne étant choisi comme limite pour les mesures car plus nettement visible sur les coupes.

\section{B. - Animaux et traitements}

Deux expériences ont été faites pour connaître la croissance folliculaire : dans les jours suivant l'injection d'une dose élevée de PMSG (exp. A) et à plus long terme après injections répétées de faibles doses de PMSG (exp. B).

\section{Expérience $A$.}

I 2 veaux reçoivent une injection de 2 ooo UI de PMSG et sont abattus $8,12,16,24,48$ ou 72 heures après.

\section{Expérience $B$.}

24 veaux reçoivent $3,4,5$ ou 8 injections de I25 UI de PMSG espacées de 2 jours. Tous sont porteurs d'une éponge vaginale qui est imprégnée (B-FGA) ou non (B-o) du progestagène. On a ainsi composé 8 lots de 3 veaux, abattus 2 jours après la dernière injection gonadotrope, soit 6,8 , ro ou I6 jours après le début du traitement. Un veau du lot FGA-ro jours a été éliminé, n'ayant pas de gonades.

\section{Matériel.}

On a utilisé des veaux Normands nourris exclusivement au lait artificiel. Leur âge n'est pas connu, mais leur poids (I 5 à I $49 \mathrm{~kg}$ lors de l'abattage) indique qu'ils sont âgés de 4 mois environ.

La PMSG ("Intervet") est injectée par voie intraveineuse à $9 \mathrm{~h}$ zo le matin pour l'expérience $\mathrm{B}$ et à divers moments dans la journée pour l'expérience $\mathrm{A}$.

Les éponges vaginales, du format couramment utilisé pour la synchronisation de l'œstrus des brebis, étaient imprégnées ou non avec $60 \mathrm{mg}$ d'acétate de fluorogestone ("Clin-Byla ") et posées en même temps que la première injection de PMSG. Elles étaient maintenues en place jusqu'à l'abattage (ces éponges placées derrière l'hymen n'étaient jamais perdues).

\section{RÉSULTATS}

\section{I. - Approximation de la méthode d'examen des ovaires}

Le tableau I compare les effectifs folliculaires recensés par examen global des ovaires entiers et par examen sur coupes des mêmes ovaires.

Dans chaque groupe expérimental, le nombre de follicules trouvé sur coupes est supérieur (de $37 \mathrm{p}$. I00 en moyenne) pour les catégories I à 3,9 $\mathrm{mm}$ et sensiblement identique à partir de $4 \mathrm{~mm}$.

Au total, l'effectif folliculaire trouvé sur coupes est supérieur de 30 p. Ioo à celui estimé par examen des ovaires in toto.

\section{2. - Effectifs folliculaires}

La population folliculaire supérieure à I mm est d'une grande variabilité : nous avons dénombré de II à 246 follicules pour les 2 ovaires.

Notons que ces différences se rencontrent dans le même lot expérimental (ex. tab1. 4 : veau 4 I : I5 follicules ; veau $3940: 246$ follicules). 


\section{TABLEAU I}

Comparaison des effectifs folliculaives extimés après examen des ovaires in toto ou après examen des coupes

\begin{tabular}{|c|c|c|c|c|c|c|c|c|c|}
\hline \multirow{2}{*}{ Lots } & \multirow{2}{*}{$\begin{array}{c}\text { Nombre } \\
\text { d'animaux }\end{array}$} & \multirow{2}{*}{$\begin{array}{l}\text { Mode de } \\
\text { recensement }\end{array}$} & \multicolumn{7}{|c|}{$\begin{array}{l}\text { Effectifs folliculaires moyens par classes de diametre } \\
\qquad(\mathrm{mm})\end{array}$} \\
\hline & & & $1-1,9$ & $2-2,9$ & $3-3,9$ & $4-4,9$ & $5-5,9$ & $\geqslant 6$ & Total \\
\hline Exp. B-0 & 12 & $\begin{array}{l}\text { ovaires in toto } \\
\text { coupes }\end{array}$ & $\begin{array}{l}31,5 \\
36,4\end{array}$ & $\begin{array}{l}28,9 \\
58,3\end{array}$ & $\begin{array}{l}10,5 \\
11,2\end{array}$ & $\begin{array}{l}2,7 \\
1,9\end{array}$ & $\begin{array}{l}0,7 \\
0,7\end{array}$ & $\begin{array}{l}2,0 \\
1,8\end{array}$ & $\begin{array}{r}76,3 \\
110,3\end{array}$ \\
\hline Exp. B-FGA & 11 & $\begin{array}{l}\text { ovaires in toto } \\
\text { coupes }\end{array}$ & $\begin{array}{l}32,7 \\
15,4\end{array}$ & $\begin{array}{l}20,2 \\
46,0\end{array}$ & $\begin{array}{r}6,7 \\
15,7\end{array}$ & $\begin{array}{l}2,3 \\
1,7\end{array}$ & $\begin{array}{l}0,3 \\
0,2\end{array}$ & $\begin{array}{l}1,0 \\
1,0\end{array}$ & $\begin{array}{l}63,2 \\
80,0\end{array}$ \\
\hline Exp. A & 12 & $\begin{array}{l}\text { ovaires in toto } \\
\text { coupes }\end{array}$ & $\begin{array}{l}22,1 \\
16,1\end{array}$ & $\begin{array}{l}17,8 \\
36,5\end{array}$ & $\begin{array}{r}9,7 \\
13,3\end{array}$ & $\begin{array}{l}4,0 \\
5,1\end{array}$ & $\begin{array}{l}1,5 \\
1,6\end{array}$ & $\begin{array}{l}3,6 \\
1,9\end{array}$ & $\begin{array}{l}58,7 \\
74,5\end{array}$ \\
\hline Tous & 35 & $\begin{array}{l}\text { ovaires in toto } \\
\text { coupes }\end{array}$ & & $\begin{array}{l}60,0 \\
83,1\end{array}$ & & & $\begin{array}{l}6,1 \\
5,7\end{array}$ & & $\begin{array}{l}66,1 \\
88,8\end{array}$ \\
\hline
\end{tabular}

TABLEAU 2

Comparaison des effectifs folliculaires des ovaires droit et gauche

\begin{tabular}{|c|c|c|c|c|c|}
\hline \multirow{3}{*}{ Lots } & \multirow{3}{*}{$\begin{array}{l}\text { Nombre } \\
\text { d'animaux }\end{array}$} & \multicolumn{4}{|c|}{$\begin{array}{l}\text { Effectifs folliculaires moyens par classes } \\
\text { de diametre }(\mathrm{mm})\end{array}$} \\
\hline & & \multicolumn{2}{|c|}{$1-3,9$} & \multicolumn{2}{|c|}{$\geqslant 4$} \\
\hline & & $\mathrm{OG}$ & OD & OG & OD \\
\hline Exp. B-0 & 12 & $\mathbf{5 5 , 9}$ & 49,8 & 1,8 & 2,7 \\
\hline Exp. B-FGA & 11 & 37,9 & 39,3 & 1,3 & 1,5 \\
\hline Exp. A & 12 & 33,2 & 32,6 & 4,9 & 3,7 \\
\hline Tous & 35 & 42,3 & 40,5 & 2,7 & 2,7 \\
\hline
\end{tabular}

Le tableau 2 compare les effectifs folliculaires des ovaires droit et gauche : on trouve en moyenne 45,0 follicules supérieurs à I mm sur l'ovaire gauche et 43,2 sur l'ovaire droit.

\section{3. - Réponses ovariennes à deux types de traitement de PMSG (expérience $\mathrm{A}$ et expérience B-o)}

Le tableau 3 montre l'évolution de la population folliculaire après l'injection de 2000 UI de PMSG (exp. A). 8 et I 2 h après cette injection, l'effectif folliculaire par classe de taille n'est pas modifié, la presque totalité des follicules reste d'un dia- 
RÉPONSE FOL,LICULAIRE DU VEAU A PMSG

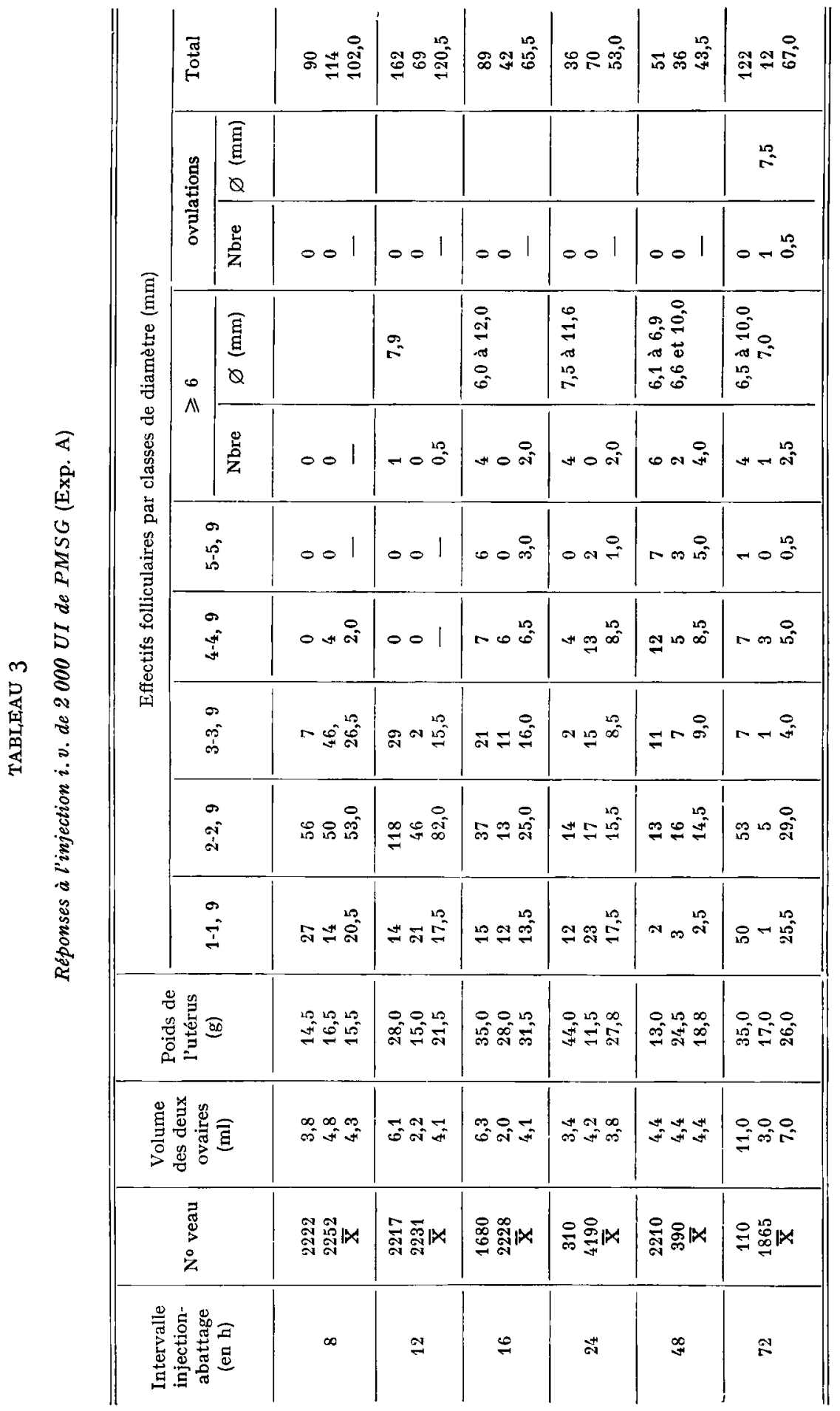




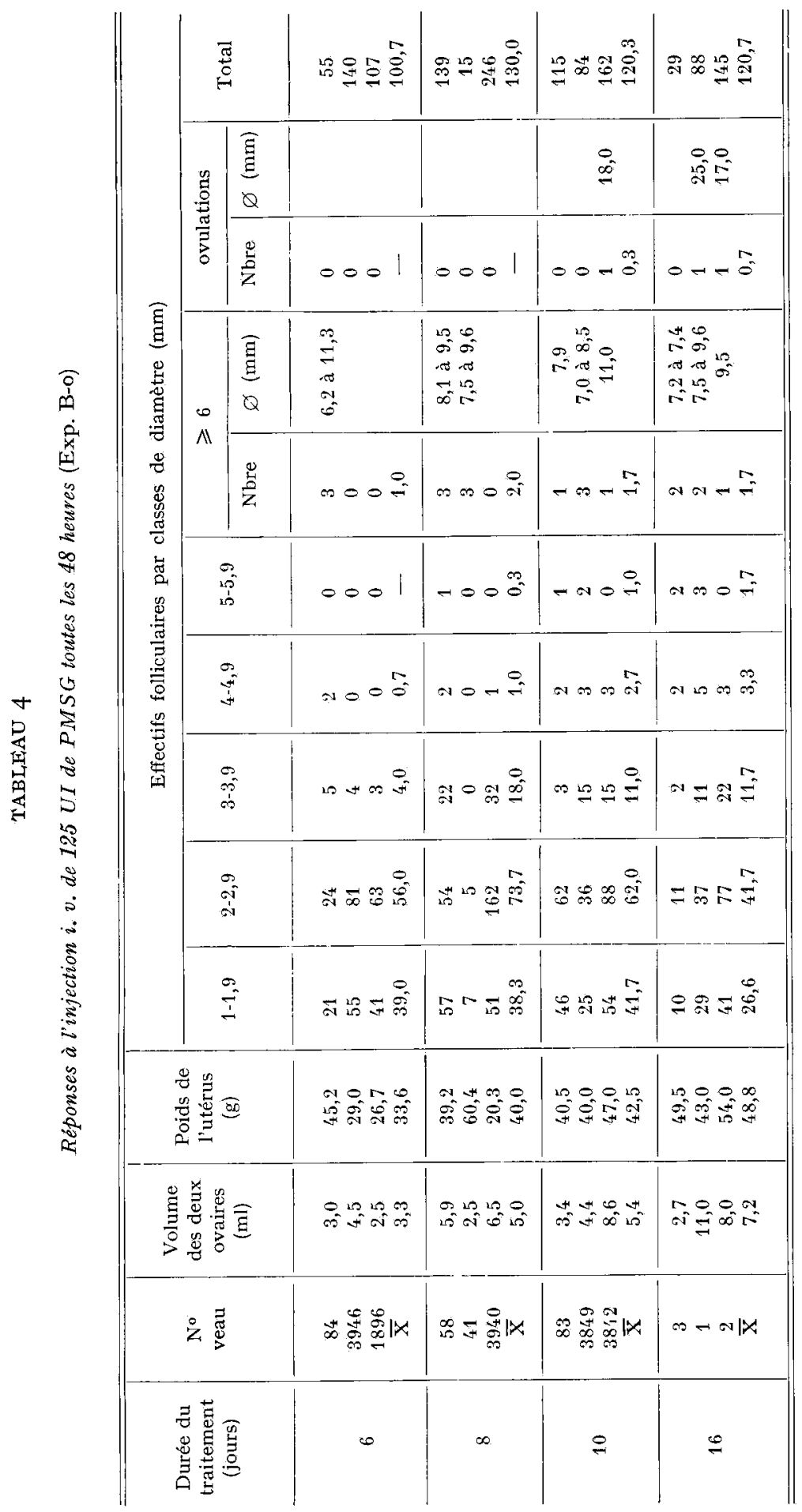




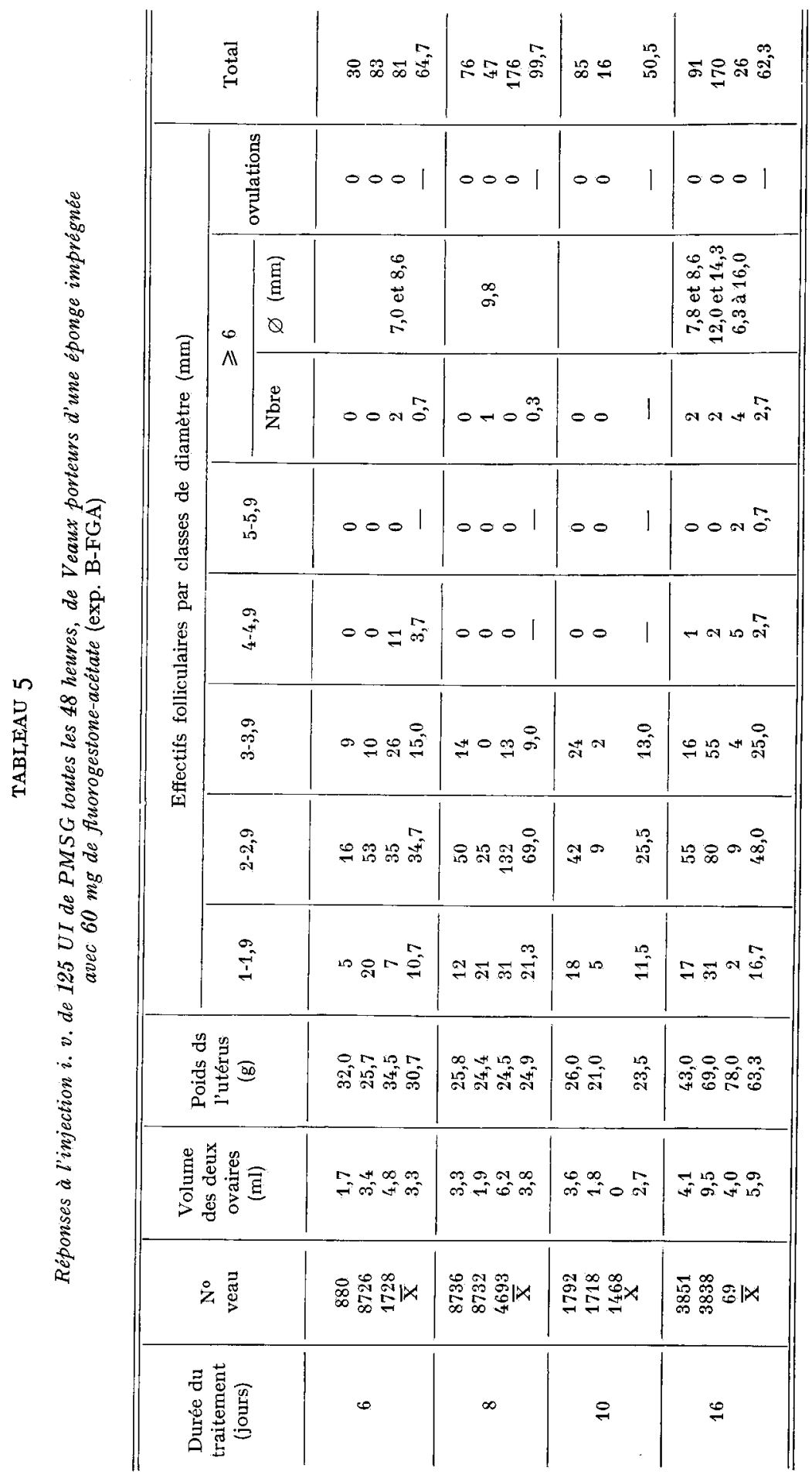


mètre inférieur à $4 \mathrm{~mm}$. A partir de $\mathrm{I} 6 \mathrm{~h}$ après l'injection de PMSG, on rencontre toujours des follicules stimulés dont le diamètre dépasse $4 \mathrm{~mm}$ et souvent $6 \mathrm{~mm}$. La figure I exprime nettement cette évolution en pourcentage de follicules stimulés $(\geqslant 4 \mathrm{~mm})$ par rapport à la population totale des 2 ovaires.

On remarque (tabl. 3) que les plus gros follicules ne dépassent pas Io à I2 $\mathrm{mm}$ et que l'ovulation peut se produire (veau I865) dans les $72 \mathrm{~h}$ après l'injection, le follicule rompu étant de taille réduite $(7,5 \mathrm{~mm})$.
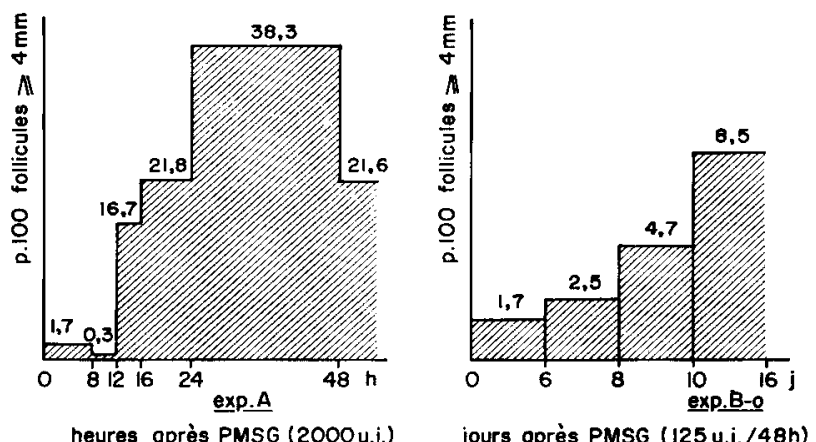

Fig. I. - Evolution dans le temps des follicules de $4 \mathrm{~mm}$ après 2 types de traitements gonadotropes (exp. A et B-o)

Après un traitement prolongé d'un faible apport gonadotrope entretenu durant 6 à I6 jours (exp. B-o), on constate une évolution de la population folliculaire qui n'est pas sans analogie avec celle de l'expérience $A$. Après 6 jours de traitement, on dénombre, comme dans le lot " $8 \mathrm{~h}$ " de l'expérience A, I,7 p. Ioo des follicules ayant un diamètre minimum de $4 \mathrm{~mm}$, puis cet effectif croît avec le temps pour atteindre 8,5 p. Ioo à 16 jours (fig. I). Cependant, contrairement à l'expérience $\mathrm{A}$, ce mode de traitement permet d'obtenir, depuis ro jours, de gros follicules rompus de I7 à $25 \mathrm{~mm}$, les follicules inférieurs à $\mathrm{I} 2 \mathrm{~mm}$ n'ayant pas ovulé, même après I6 jours de traitement (tabl. 4).

\section{4. - Influence de FGA sur la réponse ovarienne à PMSG (expérience B-FGA)}

$\mathrm{Si}$, en même temps qu'on répète toutes les $48 \mathrm{~h}$ les injections de PMSG, on laisse agir une éponge vaginale imprégnée de $\mathrm{FGA}$, on constate qu'il n'y a pas de croissance folliculaire (tabl. 5) jusqu'au $\mathrm{ro}^{\mathrm{e}}$ jour de traitement. Cependant cette action n'est pas retrouvée après I6 jours, puisque tous les animaux ont des follicules supérieurs à $6 \mathrm{~mm}$, pouvant atteindre $\mathrm{I} 4$ à $\mathrm{I} 6 \mathrm{~mm}$, mais aucun de ces veaux n'a ovulé.

\section{5. - Influence de la stimulation folliculaire sur le poids utérin}

Les tableaux 3, 4 et 5 indiquent le volume des deux ovaires et le poids de l'utérus pour chaque animal. 


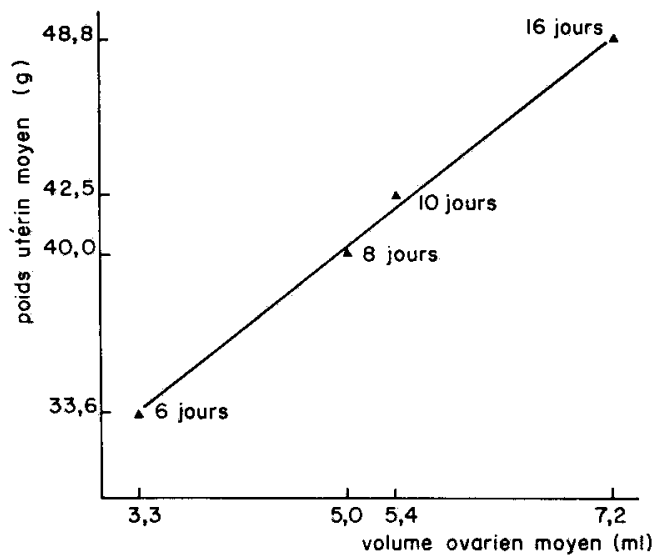

FIG. 2. - Accroissement comparé du poids utérin et du volume ovarien (exp. B-o)

Aucune relation ne peut être montrée entre ces deux variables pour l'expérience $\mathrm{A}$ (temps courts), mais, malgré la variabilité individuelle, la figure 2 montre que le poids utérin moyen augmente dans l'expérience B-o en fonction de la durée du traitement et/ou du volume ovarien. Le tableau 6 , mêlant les expériences B-o et B-FGA, montre que le poids utérin est fonction du développement folliculaire. Il est en moyenne de 25,6 g s'il n'y a pas de follicules de $6 \mathrm{~mm}$ sur l'ovaire et de $48,3 \mathrm{~g}$ dans l'autre cas.

\section{TABLEAU 6}

Relation poids utérin-Présence de gros follicules

Exp. B-o et B-FGA

\begin{tabular}{|c|c|c|c|c|c|c|}
\hline \multirow{2}{*}{\multicolumn{2}{|c|}{$\begin{array}{l}\text { Présence de follicules } \\
\geqslant 6 \mathrm{~mm} \text { sur les ovaires } \\
\text { à l'abattage }\end{array}$}} & \multicolumn{5}{|c|}{$\begin{array}{l}\text { Poids utérin moyen selon la durée du traitement en jours } \\
\text { (Nbre animaux) }\end{array}$} \\
\hline & & 6 & 8 & 10 & 16 & Total \\
\hline 0 & $\begin{array}{l}\text { PMSG seule } \\
\text { PMSG }+ \text { FGA }\end{array}$ & $\begin{array}{l}27,8(2) \\
28,8(2)\end{array}$ & $\begin{array}{l}20,3(1) \\
25,1(2)\end{array}$ & $23,5(2)$ & & $25,6(9)$ \\
\hline+ & $\begin{array}{l}\text { PMSG seule } \\
\text { PMSG }+ \text { FGA }\end{array}$ & $\begin{array}{l}45,2(1) \\
34,5(1)\end{array}$ & $\begin{array}{l}49,8(2) \\
24,4(1)\end{array}$ & $42,5(3)$ & $\begin{array}{l}48,8(3) \\
63,3(3)\end{array}$ & $48,3(14)$ \\
\hline
\end{tabular}

\section{DISCUSSION}

Io La technique d'examen en coupes sériées épaisses permet d'obtenir rapidement une bonne estimation de la population folliculaire supérieure à $\mathrm{I} \mathrm{mm}$. On dénombre, en moyenne, $30 \mathrm{p}$. Ioo de follicules supplémentaires par rapport aux résul- 
tats du comptage sur l'ovaire in toto. En effet, la section des ovaires permet de découvrir de petits follicules non visibles sur la surface de l'organe entier. Cependant, toutes les mesures étant effectuées par défaut (voir méthodes d'étude) il y a sousestimation de la population totale, par sous-évaluation des effectifs des catégories inférieures, ce qui autorise toutefois une étude comparative.

L'effectif total des follicules supérieurs à I mm est d'une grande variabilité et n'augmente pas après chacun des traitements. SETTERGREEN (I964) a montré la fréquence des hypoplasies gonadiques partielles chez les Bovins, et HowE et al. (I962) mettent en rapport cette variabilité avec les cycles de croissance et régression folliculaires observés par laparotomies répétées de veaux impubères.

Les effectifs de follicules à antrum des ovaires droit et gauche sont semblables ce qui confirme les résultats de RAJAKOSKI (I960) et SETTEERGREEN (I964).

$2^{\circ}$ L'analyse des résultats des expériences $\mathrm{A}$ et $\mathrm{B}-\mathrm{o}$ (tabl. 3 et 4 ; fig. I et 3 ) montre que la stimulation folliculaire (par PMSG) augmente l'effectif des follicules supérieurs à $4 \mathrm{~mm}$. La classe 4 à $4,9 \mathrm{~mm}$ est la première à répercuter l'action gonado-
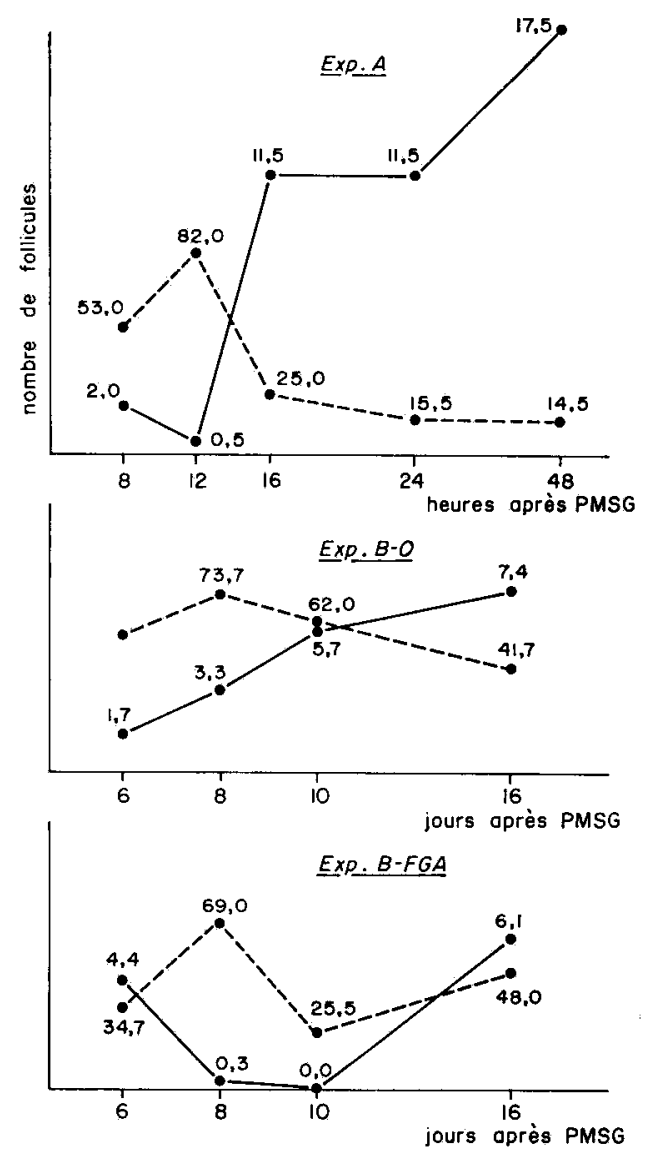

Fig. 3. - Evolution comparée des effectifs de follicules dans les classes 2-2, $9 \mathrm{~mm}$ et $\geqslant 4 \mathrm{~mm}$ classe $2-2,9 \mathrm{~mm}$ classe $\geqslant 4 \mathrm{~mm}$ 
trope ; elle constitue ainsi la taille critique puisque c'est à partir d'elle que s'accroîtra le nombre des plus gros follicules.

$3^{\circ}$ Cette classe de population augmente $16 \mathrm{~h}$ après injection de 2 ooo UI de PMSG (exp. A) ou 8 jours après l'injection de I25 UI de PMSG toutes les 48 heures (exp. B-o).

$4^{\circ}$ La classe 2 à $2,9 \mathrm{~mm}$ est toujours la plus riche et constitue la réserve d'où échappent les follicules de taille critique (4 à 4,9 mm).

En effet (fig. 3 et tabl. 3) l'effectif moyen de cette classe qui est de 53 à 82 respectivement 8 et I 2 heures après l'injection de 2 ooo UI de PMSG diminue à 25, I5,5 et 14,5 respectivement après $I 6,24$ et 48 heures ; en même temps, l'ensemble des follicules supérieurs à $4 \mathrm{~mm}$ augmente ses effectifs de 2 et 0,5 après 8 et I 2 heures à II, 5 , II, 5 et I7,5 après I6, 24 et 48 heures. Cette évolution est moins sensible dans le traitement long (B-0, tabl. 4), vraisemblablement parce que la catégorie stimulée $(\geqslant 4 \mathrm{~mm}$ ) compte toujours des effectifs faibles : $I, 7$ après 6 jours contre $3,3,5,3$ et 6,7 respectivement après 8 , no et 16 jours.

$5^{\circ}$ En présence du progestagène (B-FGA, tabl. 5) l'importance de la classe 2 à $2,9 \mathrm{~mm}$ n'évolue pas : 34,7 follicules après 6 jours de traitement contre 69,0 et 25,5 respectivement après 8 et Io jours. Or, les catégories supérieures à $4 \mathrm{~mm}$ n'évoluent pas non plus dans le même temps, ce qui confirme la fonction de stockage de la classe 2 à $2,9 \mathrm{~mm}$.

$6^{\circ}$ Les classes de follicules intermédiaires (I à $1,9 \mathrm{~mm}$ et 3 à $3,9 \mathrm{~mm}$ ) si elles montrent des variations en rapport avec l'effectif folliculaire total de chaque animal, évoluent peu quel que soit le traitement. Ainsi, on peut les considérer comme des classes de passage par opposition à la classe $2-2,9 \mathrm{~mm}$ qui est une classe de stockage et à la classe 4-4,9 qui est la classe critique (début de croissance des follicules stimulés par l'hormone exogène).

Il est évident que le choix arbitraire de nos limites de classe ne peut que rendre imparfaitement compte de 1'ensemble du phénomène ; cependant le tableau 7 reflète cette évolution.

\section{TABLEAU 7}

Évolution de la population folliculaire lors de la croissance des follicules supérieurs à $4 \mathrm{~mm}$ (tous traitements)

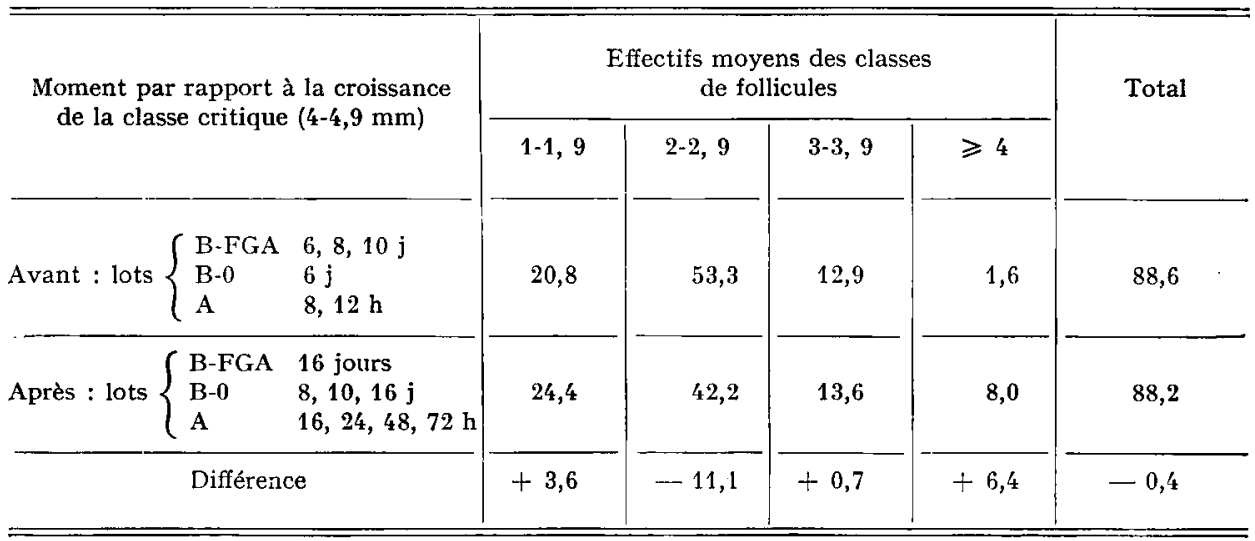


$7^{\circ}$ Le « pompage » de follicules dans la classe $2-2,9$, permettant la croissance de la classe critique, ne semble pas compensé par un apport immédiat à partir des classes inférieures à I mm. Cela pourrait rendre compte des limites constatées quant aux résultats des traitements de superovulation chez les Bovins, quelle que soit la dose gonadrotrope administrée, 1 'importance de la réponse de chaque femelle étant ici fonction de son effectif folliculaire dans la classe $2-2,9 \mathrm{~mm}$, avant le traitement.

$8^{\circ}$ Le rendement du passage de la classe $2-2,9 \mathrm{~mm}$ à la classe $4-4,9 \mathrm{~mm}$ et audessus peut être estimé (tabl. 7).

Pour l'ensemble des traitements, moins de 2 follicules sur 3 de la classe $2-2,9 \mathrm{~mm}$ poursuivent leur croissance. La faible augmentation de l'effectif dans la classe I-I,9 mm $(+3,6)$ reflète peut-être les atrésies subies par les follicules de dimension supérieure, puisque la population totale n'augmente pas.

$9^{\circ}$ On obtient l'ovulation des animaux, soit ro jours après le début d'un faible apport gonadotrope entretenu (exp. B-o), soit 3 jours après l'injection unique d'une forte dose (exp. A). On remarque que dans les deux cas un seul follicule subit 1'ovulation ; ce follicule devant être (voir introduction) le plus gros préexistant sur l'ovaire au début du traitement, il est possible que sa présence (sécrétion d'œstrogènes) ou son évolution en corps jaune (sécrétion de progestérone) empêche l'ovulation d'autres follicules en l'absence d'une hormone ovulante exogène.

Io ${ }^{\circ}$ Dans le cas d'un apport gonadotrope entretenu, associé à un traitement progestagène (exp. B-FGA), le blocage de la croissance folliculaire jusqu'à ro jours (on ne retrouve pas nettement la régression folliculaire constatée par O'BRIEN et MILLER, r970, chez 1'Agnelle) est suivi d'une croissance accélérée. On peut penser qu'après Io jours 1'éponge est épuisée en FGA, mais 1'apparition en quelques jours de follicules de grande taille (jusqu'à $\mathrm{I} 6 \mathrm{~mm}$ ) ne correspond pas à ce qui est vu après un traitement de 6 jours avec la PMSG seule (exp. B-o) et doit résulter d'un effet " rebound " de décharge par 1'hypophyse de la LH stockée.

I I ${ }^{\circ}$ Le traitement gonadotrope prolongé permet d'obtenir la croissance de l'utérus de roo p. Ioo. Cet effet, croissant dans le temps, a pour médiateur la sécrétion folliculaire (tabl. 6) ce qui indique que l'utérus du Veau impubère est sensible au taux d'œestrogènes endogènes. Un tel développement utérin devrait être bénéfique à l'accomplissement de la fécondation et à la segmentation.

Rę̧u pour publication en janvier 1972.

\section{SUMMARY}

FOLIICULAR RESPONSE OF THE IMMATURE FEMALE CALF TO VARIOUS

SERIC GONADOTROPIN TREATMENTS, ASSOCIATED OR UNASSOCIATED WITH A PROGESTIN

After various hormone treatments, immature calf follicles at least $\mathbf{I} \mathrm{mm}$ in diameter were measured and counted from seriated cross-sections of $25^{\circ} \mu$ thickness obtained with a cryostat.

The counting method used is rapid and shows $30 \mathrm{p}$. I oo supplementary follicles as compared to in toto ovary examination. The follicle population varies widely, but is not different for the two ovaries. 
Follicular growth following intravenous injection of 2000 I U PMSG (Experiment A) or intravenous injections of I 25 I U PMSG every 2 days (Experiment B) is first noticed in $4 \mathrm{~mm}$ follicles.

4-5 $\mathrm{mm}$ follicles constitute the critical population, the number of which increases 16 hours (Experiment $\mathrm{A}$ ) or 8 days (Experiment B) after the beginning of the treatment at the expense of $2-3 \mathrm{~mm}$ follicles which constitute the storage population.

Ovulation of follicles stimulated by PMSG alone is obtained after 72 hours (Experiment A) or ro days (Experiment B), but only prolonged treatment (Experiment B) allows differenciation of normal corpora lutea (I 7-25 mm) and Ioo p. Ioo uterus growth.

Using a vaginal sponge impregnated with $60 \mathrm{mg}$ of FGA at the beginning of the long PSMG treatment (Experiment B-FGA) forestalls any follicular evolution up to ro days, and after that time there is rapid growth of several follicles.

\section{RÉFÉRENCES BIBLIOGRAPHIQUES}

Avery T. L., Fahning M. L., Graham E. F., I962. Investigations associated with the transplantation of bovine ova. II. Superovulation . J. Reprod, Fertil., 3, 212-217.

Black W. G., Ulberg L. C., Christian R. E., Casida L. E., r953. Ovulation and fertilization in the hormone-stimulated calf. J. Dairy Sci., 36, 274.

Casida L. E., Chapman A. B., Ruper I. W., I935. Ovarian development in calves. Austr. J. Agric. Res., 50, 953 .

Casida L. E., Meyer R. K., Mc Shan W. H., Wisnicky W., I943. Effects of pituitary gonadotrophins on the ovaries and the induction of superfecundity in cattle. Amer. J. Vet. Res., 6, 76-94.

ERIckson B. H., I966. Development and senescence of the postnatal bovine ovary. J. anim. Sci., 25, 3, 800-805.

HAFE2 E. S. E., I970. Superovulation and preservation of mammalian eggs. Acta Endocr., 62, suppl$140,44 \mathrm{p}$.

Howe G. R., Black D. L., Foley R. C., Black W. G., I962. Ovarian activity in prepuberal dairy calves. J. anim. Sci., 21, 82 .

Jainudeen M. R., Hafez E. S. E., Lineweaver J. A., I966. Superovulation in the calf. $J$ Reprod. Fertil., 12, 149-I53.

Lineweaver J. A., HAFez E. S. E., I970. Ovarian responses in gonadotropin-treated calves. Amer. J. Vet. Res., 31, 2I57-2166.

MaRden W. G. R., I953. The hormone control of ovulation in the calf, J. Agric. Sci., 43, 381-416

Mauléon P., Mariana J.-C., Benoit M., Solari A., Chupin D., I97o. Influence de différentes doses de PMSG et HCG injectées en phase folliculaire du cycle œstrien sur le nombre et le rendement d'ovulations de Vaches de race Française Frisonne Pie Noire. Ann. Biol. anim. Bioch. Biophys., 10, $\mathrm{n}^{\circ}$ hors-série I, $3 \mathrm{I}-46$.

O'Brien C. A., Miller C. F., I97o. Response of fattening ewe lands to oral melengestrol acetate. J. anim. Sci., 31, 3 .

Onuma H., HahN J., Maurer R. R., Foote R. H., I969. Repeated super-ovulation in calves. J. anim. Sci., 28, 5, 634-637.

ONuma H., Foote R. H., 1969. Superovulation in prepuberal calves on two levels of nutrient intake. J. anim. Sci., 28, 6, 771-774.

Onuma H., Hahn J., Foote R. H., r97o. Factors affecting superovulation, fertilization and recovery of superovulated ova in prepuberal cattle. J. Reprod. Fertil., 21, II9-126.

Pedersen T., rg7o. Determination of follicle growth rate in the ovary of the immature mouse. J. Reprod. Fertil., 21, 81.

RAjAkoski E, I960. The ovarian follicular system in sexually mature heifers with special reference to seasonal, cyclical and left-right variations. Acta Endocr., 34, suppl. 52.

Seidel G. E., Foote R. H., 1969. Superovulatory response in calves zero, 4 and 8 weeks old. $J$ Dairy Sci., 52, 6, 905 .

Seidel G. E., Larson L. L,, Foote R. H., ig7r. Effects of age and gonadotropin treatment on superovulation in the calf. J. anim. Sci. 33, 3,6I7-622.

SetTergreen 1., 1964. The ovarian morphology in clinical bovine gonadal hypoplasia with some aspects of its endocrine relations, Acta Vet. Scand., 5, suppl., r, ro7 p.

YING S. Y., MEYER R. K., I969. Dose dependent pregnant mare'serum induced ovulation in immature rats .Proc. Soc. exp. Biol. Med., 130, 40-54. 